\title{
MONOCHROMATIC CCD IMAGES OF THREE PLANETARY NEBULAE
}

\author{
WALTER A. FEIBELMAN \\ NASA-Goddard Space Flight Center, Lab. for Astronomy and Solar Physics, Greenbelt
}

We present monochromatic false-color images of three planetary nebulae obtained by means of a CCD camera at the $\mathrm{f} / 13.5$ focus of the $0.91 \mathrm{~m}$ reflector of the Cerro Telolo Interamerican Observatory in August, 1985. The resultant image scale is 0.363 arcsec square per pixel. The objects, IC 1297, NGC 7009, and M2-9 were imaged through narrow band filters in the $\mathrm{V}$ band, [O III] $\lambda 5007$, and $\mathrm{H} \alpha \lambda 6563$, respectively. The $\mathrm{V}$ band filter corresponds to the standeard Johnson V magnitude. The $\lambda 5007$ filter has a FWHM of $14 \AA$. The $\mathrm{H} \alpha$ filter has a FWHM of $73 \AA$, thus including contributions from the [N II] $\lambda \lambda 6548,6584$ lines. IC 1297 shows a double shell structure with a pronounced bright rim at the extreme edge of the faint outer envelope, suggesting that it is density bounded. NGC 7009 (the "Saturn" nebula), in addition to the well-known double shell and extended $\mathrm{W}-\mathrm{W}$ ansae, also shows a faint large, circular outer halo which strongly suggests that NGC 7009 is a member of the rare class of triple-shell nebulae. The bipolar object M2-9 (the "Butterfly" nebula) shows a bright, non-stellar central core from which the wings extend in a nearly N-S direction. Condensations in the wings are seen particularly well for the [O III] $\lambda 5007$ images. The CCD images were obtained by B. Schaefer at CTIO and were processed at the Goddard Image Processing Facility. 